\title{
Simplified Quantification and Acquisition Protocol for 123I-MIBG Dynamic SPECT
}

\author{
Jing $\mathrm{Wu}^{1}$, Jean-Dominique Gallezot ${ }^{1}$, Yihuan $\mathrm{Lu}^{1}$, Qing $\mathrm{Ye}^{1,2}$, Hui Liu ${ }^{3}$, Denise A. Esserman ${ }^{4}$, Tassos C. Kyriakides ${ }^{4}$, \\ Stephanie L. Thorn ${ }^{3}$, Taraneh Hashemi Zonouz ${ }^{3}$, Yi-Hwa Liu ${ }^{3,5,6}$, Rachel J. Lampert ${ }^{3}$, Albert J. Sinusas ${ }^{1,3}$, \\ Richard E. Carson ${ }^{1}$, and Chi Liu ${ }^{1}$ \\ ${ }^{I}$ Department of Radiology and Biomedical Imaging, Yale University, New Haven, Connecticut; ${ }^{2}$ Department of Engineering Physics, \\ Key Laboratory of Particle and Radiation Imaging (Tsinghua University), Ministry of Education, Beijing, China; ${ }^{3}$ Department of \\ Internal Medicine (Cardiology), Yale University, New Haven, Connecticut; ${ }^{4}$ Yale School of Public Health (Biostatistics), Yale \\ University, New Haven, Connecticut; ${ }^{5}$ Department of Biomedical Imaging and Radiological Sciences, National Yang-Ming \\ University, Taipei, Taiwan; and ${ }^{6}$ Department of Biomedical Engineering, Chung Yuan Christian University, Taoyuan, Taiwan
}

Previous studies have demonstrated the feasibility of absolute quantification of dynamic ${ }^{123}$ I-metaiodobenzylguanidine (123I-MIBG) SPECT imaging in humans. This work reports a simplified quantification method for dynamic ${ }^{123}$ /-MIBG SPECT using practical protocols with shortened acquisition time and voxel-by-voxel parametric imaging. Methods: Twelve healthy human volunteers underwent five 15-min dynamic SPECT scans at 0, 15, 90, 120, and 180 min after ${ }^{123}$ I-MIBG injection. List-mode SPECT data were binned into 29 frames and reconstructed with corrections for attenuation, scatter, and decay. Population-based blood-to-plasma correction and metabolite correction were applied to the image-derived input function. Likelihood estimation in graphical analysis (LEGA) was used as a simplified model to obtain volume of distribution $\left(V_{T}\right)$ values, which were compared with those obtained with the reversible 2-tissue (2T) compartment model. Three simplified protocols were evaluated with 2T and LEGA using a 30-min scan started simultaneously with tracer injection plus a 15 -min scan at 90,120 , or 180 min after injection. Voxel-by-voxel LEGA fitting was applied to the aligned dynamic images using both the full protocol (five 15-min scans) and the simplified protocols. Results: Correlation analysis $\left(y=0.955 x+0.547, R^{2}=0.997\right)$ and Bland-Altman plot (mean difference, $-0.8 \mathrm{~mL} / \mathrm{cm}^{3} ; 95 \%$ limits of agreement, $[-2.5,1.0] \mathrm{mL} /$ $\mathrm{cm}^{3}$; normal $V_{\mathrm{T}}$ range, $29.0 \pm 12.4 \mathrm{~mL} / \mathrm{cm}^{3}$ ) showed that LEGA can be used as a simplified model of $2 \mathrm{~T}$ for ${ }^{123}$-MIBG. High-quality $V_{T}$ parametric images could be obtained with LEGA. Region-of-interest (ROI) modeling and parametric imaging results were in excellent agreement as determined by correlation analysis $\left(y=0.999 x-1.026, R^{2}=\right.$ 0.982 ) and Bland-Altman plot (mean difference, $-1.0 \mathrm{~mL} / \mathrm{cm}^{3} ; 95 \%$ limits of agreement, $\left.[-4.2,2.1] \mathrm{mL} / \mathrm{cm}^{3}\right) . V_{\mathrm{T}}$ correlated reasonably well between all simplified protocols and the full protocol with LEGA but not with $2 \mathrm{~T}$. The $V_{\mathrm{T}}$ results were more reliable when there was a longer interval between the 2 acquisitions in the simplified protocols. Conclusion: For ROI-based kinetic modeling and parametric imaging, reliable quantification of dynamic ${ }^{123}$ I-MIBG SPECT can be achieved with LEGA using a simplified protocol of a 30-min scan starting with tracer injection plus a 15-min scan no earlier than $180 \mathrm{~min}$ after injection.

\footnotetext{
Received Sep. 14, 2017; revision accepted Feb. 3, 2018.

For correspondence or reprints contact: Chi Liu, Department of Radiology and Biomedical Imaging, Yale University, P.O. Box 208048, New Haven, CT 06520.

E-mail: chi.liu@yale.edu

Published online Feb. 23, 2018.

COPYRIGHT (C 2018 by the Society of Nuclear Medicine and Molecular Imaging.
}

Key Words: ${ }^{123}$ I-metaiodobenzylguanidine (123|-MIBG); dynamic SPECT; simplified quantification; parametric imaging

J Nucl Med 2018; 59:1574-1580

DOI: 10.2967/jnumed.117.202143

$\mathbf{M}$ yocardial sympathetic imaging with ${ }^{123}$ I-metaiodobenzylguanidine ( ${ }^{123} \mathrm{I}-\mathrm{MIBG}$ ) has been extensively used for assessing patients with congestive heart failure, ischemic heart disease, and primary arrhythmic disease (1-4). The heart-to-mediastinum ratio calculated from 2-dimensional (2D) planar imaging has remained the conventional standard for ${ }^{123} \mathrm{I}-\mathrm{MIBG}$ quantification. However, heart-to-mediastinum ratios obtained from $2 \mathrm{D}$ planar imaging are only semiquantitative because of the inclusion of background activity in the quantification of the activity within the heart from a 2D region of interest (ROI) and the lack of corrections for attenuation and scatter. The use of 3-dimensional (3D) SPECT imaging for ${ }^{123}$ I-MIBG quantification has been applied more recently to overcome these limitations and make regional analysis of myocardial uptake feasible (5-11). However, myocardial ${ }^{123}$ I-MIBG uptake changes with time, especially in heart failure patients, with the myocardial activity decreasing continuously even after $3 \mathrm{~h}$ after injection $(12,13)$. The use of 4-dimensional (4D) dynamic SPECT imaging with tracer kinetic modeling can provide intrinsic physiologic parameters for quantifying sympathetic nerve activity, which is independent of the imaging time points and has the potential to be more sensitive and accurate than the estimation of a $2 \mathrm{D}$ or $3 \mathrm{D}$ heart-to-mediastinum ratio. This dynamic 4D approach can facilitate standardization of quantification of ${ }^{23}$ I-MIBG images for wider clinical applications.

Our group recently reported the feasibility of absolute quantification of sympathetic activity with 4D dynamic ${ }^{123}$ I-MIBG SPECT imaging using a stationary dedicated cardiac cadmium zinc telluride SPECT/CT scanner (Discovery NM/CT 570c; GE Healthcare) over a 3- to 4-h period (13). Population-based blood-to-plasma ratio correction and metabolite correction techniques were proposed and demonstrated to be feasible for tracer kinetic modeling in a healthy human population. The volume of distribution $\left(V_{\mathrm{T}}\right)$ values (range, $15.0-53.8 \mathrm{~mL} / \mathrm{cm}^{3}$; mean, $29.0 \pm 12.4 \mathrm{~mL} / \mathrm{cm}^{3}$ ) for ${ }^{123}$ I-MIBG in myocardium were reported for 12 healthy humans using a reversible 2-tissue (2T) compartment model.

However, 3-4 h of dynamic imaging may not be clinically feasible. To establish a more clinically practical quantitative approach, 
we have extended our previously developed dynamic ${ }^{123}$ I-MIBG technique (13) and investigated simplified acquisition protocols using only 2 scans and a shorter total acquisition time with a simplified model. We have investigated, for what is to our knowledge the first time, a voxel-by-voxel parametric imaging technique for analysis of ${ }^{123}$ I-MIBG SPECT studies that will enable future regional analysis of clinical $V_{\mathrm{T}}$ images, potentially providing additional information on the heterogeneity of myocardial sympathetic activity. This study used the same dataset from 12 healthy humans as was used in a previous report (13).

\section{MATERIALS AND METHODS}

\section{Study Subjects and Dynamic SPECT Image Generation}

Twelve healthy humans ( 7 male and 5 female; mean age $\pm \mathrm{SD}$, $48 \pm 13 \mathrm{y}$ ) received an intravenous bolus injection of $233 \pm 67 \mathrm{MBq}$ of ${ }^{123}$ I-MIBG (AdreView; GE Healthcare). Five 15-min dynamic SPECT scans were acquired on the Discovery NM/CT 570c scanner starting at $0,15,90,120$, and $180 \mathrm{~min}$ after injection. The subjects remained in the scanner for the first 2 scans and then left the scanner between the subsequent scans to take breaks. An unenhanced CT scan was acquired for attenuation correction at the end of the last SPECT acquisition. The study was approved by the institutional review board, and all subjects signed an informed consent form. The list-mode SPECT data from all 5 scans were binned offline into 29 frames $(15 \times 20 \mathrm{~s}$ and $14 \times 300 \mathrm{~s})$. The dynamic images were reconstructed using maximum-likelihood expectation maximization (80 iterations) with corrections for attenuation, scatter, and decay. Detailed information about the study subjects and the image reconstruction process was previously reported (13).

\section{Kinetic Modeling}

For each subject, an image-derived input function and myocardial time-activity curve were obtained by drawing 3D ROIs on the left ventricle blood pool cavity and on the left ventricle myocardium, respectively. To reduce the effect of body motion between scans, the ROIs of each scan were generated separately with the same volumes. Population-based blood-to-plasma ratio correction and metabolite correction, which were previously found feasible for a healthy human population (13), were applied to the image-derived input function for tracer kinetic modeling. $2 \mathrm{~T}$ was used, which has been previously identified as the optimal model for ${ }^{123}$ I-MIBG modeling $(12,13)$. However, the implementation of $2 \mathrm{~T}$ is computationally intensive, particularly for the voxel-by-voxel fitting required to generate parametric images. $2 \mathrm{~T}$ is also sensitive to the noise in the dynamic images. A simplified model is often applied to address these issues. The commonly used Logan graphical analysis (14) can estimate $V_{\mathrm{T}}$ more rapidly and robustly than $2 \mathrm{~T}$ but suffers from noise-induced bias $(15,16)$. To overcome this limitation, we used likelihood estimation in graphical analysis (LEGA) $(17,18)$, as previously implemented (19), which can robustly estimate $V_{\mathrm{T}}$ by accounting for the noise in the original data domain.

The estimated myocardial time-activity curve using LEGA, $\overline{C_{\mathrm{T}}(t)}$, can be described as follows:

$$
\begin{aligned}
\widehat{C_{\mathrm{T}}(t)} & =\beta C_{\mathrm{P}}(t) \otimes^{*} e^{-\alpha t} \\
+ & \left(\beta \int_{0}^{t^{*}} C_{\mathrm{P}}(\tau) d \tau-\alpha \int_{0}^{t^{*}}\left(C_{\mathrm{SPECT}}(\tau)-V_{\mathrm{B}} C_{\mathrm{wb}}(\tau)\right) d \tau\right) e^{-\alpha\left(t-t^{*}\right)}, \\
& t>t^{*} .
\end{aligned}
$$

$\beta$ and $\alpha$ are 2 intermediate parameters, defined by $\alpha=-1 / b$, where $b$ is the $y$-intercept of the Logan plot and $V_{\mathrm{T}}=\frac{\beta}{\alpha} \cdot t^{*}$ is the time after which the LEGA equation holds (i.e., when the Logan plot is considered linear). $\otimes^{*}$ is a customized convolution operator using only data after $t^{*}$ (i.e., $\left.f \otimes^{*} g=\int_{t^{*}}^{t} f(\tau) g(t-\tau) d \tau\right)$. $C_{\mathrm{P}}(t)$ is the plasma time-activity curve after metabolite correction used as the input function. $C_{\mathrm{wb}}(t)$ is the whole-blood time-activity curve. $V_{\mathrm{B}}$ is the blood volume term accounting for the spill-in effect from the blood pool to the myocardium. $C_{\mathrm{SPECT}}(t)$ is the myocardial time-activity curve obtained from the SPECT images, including the blood volume spill-in. $t^{*}$ was selected to be $7.5 \mathrm{~min}$ through a pilot investigation using multiple values applied on the data for 12 subjects, and $V_{\mathrm{B}}$ was set to be the mean value $0.3 \mathrm{~mL} / \mathrm{cm}^{3}$ obtained from $2 \mathrm{~T}$ (13).

The fitted parameters $V_{\mathrm{T}}$ and $\alpha$ were estimated using weighted least squares with a Marquardt-Levenberg algorithm (20) as follows:

$$
\begin{gathered}
\left(\widehat{V_{\mathrm{T}}}, \hat{\alpha}\right)=\operatorname{argument} \text { of the minimum } \sum_{i=k}^{n} w_{i}\left\{C_{\mathrm{SPECT}}\left(t_{i}\right)-\widehat{C_{\mathrm{SPECT}}}\left(t_{i}\right)\right\}^{2}, \\
\text { Eq. } 2 \\
\text { where } \widehat{C_{\mathrm{SPECT}}}\left(t_{i}\right)=\frac{1}{t_{i, \text { end }}-t_{i, \text { start }}} \int_{t_{i, \text { sart }}}^{t_{i, \text { end }}}\left(\widehat{C_{\mathrm{T}}(\widehat{t})}+V_{\mathrm{B}} C_{\mathrm{wb}}(t)\right) d t .
\end{gathered}
$$

$\widehat{C_{\mathrm{SPECT}}} \widehat{\widehat{A}}\left(t_{i}\right)$ is the estimation of $C_{\mathrm{SPECT}}(t) \cdot t_{i, \text { start }}$ and $t_{i, \text { end }}$ are the start time and end time of frame $i . k$ is the start frame number (i.e., $t_{k, \text { start }}=t^{*}$ ), and $n$ is the end frame number (i.e., the total number of frames). $w_{i}$ is the weighting factor of frame $i$ calculated by:

$$
w_{i}=\frac{\left(t_{i, \text { end }}-t_{i, \text { start }}\right)^{2}}{C_{\text {Proj }, i} \times \mathrm{DCF}_{i}^{2}},
$$

where $C_{\mathrm{Proj}, i}$ is the total projection count number of frame $i$ and $\mathrm{DCF}_{i}$ is the decay correction factor of frame $i$.

\section{Simplified Acquisition Protocols}

In clinical practice, it is often difficult to implement dynamic ${ }^{123} \mathrm{I}-$ MIBG imaging that incorporates five 15-min scans over 3-4 h. To reduce the number of scans and the total acquisition time, we investigated 3 different simplified acquisition protocols that make use of fewer scans (2 scans) and a shorter total acquisition time (45 min). Accordingly, a 30-min scan starting immediately after ${ }^{123}$ I-MIBG injection (including two 15-min scans starting at 0 and $15 \mathrm{~min}$ ) was incorporated with a 15 -min late-phase scan at $180 \mathrm{~min}(30+180 \mathrm{~min})$, $120 \mathrm{~min}(30+120 \mathrm{~min})$, or $90 \mathrm{~min}(30+90 \mathrm{~min})$ after injection.

For the full protocol with all five 15-min scans, the SPECT data were binned into 29 frames $(15 \times 20 \mathrm{~s}$ and $14 \times 300 \mathrm{~s})$. For the simplified acquisition protocols, the data of the first 30-min scan were binned into 20 frames $(15 \times 20 \mathrm{~s}$ and $5 \times 300 \mathrm{~s})$ and those of the latephase 15 -min scan were binned into 3 frames $(3 \times 300 \mathrm{~s})$. We investigated these 3 simplified protocols using both $2 \mathrm{~T}$ and LEGA.

\section{Parametric Imaging}

To establish methods to measure regional $V_{\mathrm{T}}$ to evaluate the heterogeneity of ${ }^{123} \mathrm{I}-\mathrm{MIBG}$ uptake in the myocardium, parametric imaging was implemented with voxel-by-voxel modeling on the dynamic SPECT images. For parametric imaging, only LEGA was used in this work, because $2 \mathrm{~T}$ is time-consuming and sensitive to noise on the voxel level. Images were registered before the voxel-by-voxel fitting, because the subject left the scanner between scans. To minimize the registration error due to the high noise and the truncation artifact in the SPECT image, we implemented the registration based on the CT-derived attenuation maps instead of SPECT images. First, the attenuation map was registered to each SPECT image (without attenuation correction) on a GE Healthcare Xeleris workstation using the 
attenuation correction quality control protocol. The 15-min SPECT scan starting at $15 \mathrm{~min}$ after injection was chosen as the reference scan. The attenuation map of each scan was rigidly registered to that of the reference scan using Biolmage Suite software with the normalized mutual information as the similarity metric (21). Then, the transformation matrix derived from attenuation map registration was applied to the SPECT images of each scan to register all the dynamic SPECT images to the reference scan. Voxel-by-voxel fitting was implemented on the aligned dynamic SPECT images using LEGA. The input function used for parametric imaging was the same as that used in the ROI-based modeling process. The simplified protocols were also investigated in the parametric imaging study.

\section{Statistical Methods}

Correlation values and least-squares fits were calculated for the 12 healthy humans, first, between ROI $V_{\mathrm{T}}$ with $2 \mathrm{~T}$ and with LEGA; second, between ROI $V_{\mathrm{T}}$ and mean myocardial $V_{\mathrm{T}}$ using parametric imaging where both were derived using LEGA; and third, between ROI $V_{\mathrm{T}}$ with the simplified protocols (with both 2T and LEGA) and the full protocol with $2 \mathrm{~T}$ (denoted as the gold standard). Bland-Altman analysis (22) was also used to further investigate the agreement of the methods.

On parametric images, the normalized root-mean-squared error (NRMSE) of the difference in myocardial $V_{\mathrm{T}}$ between the simplified and full protocols was calculated as

$$
\text { NRMSE }=\frac{\sqrt{\sum_{i=1}^{N}\left(x_{i}^{\prime}-x_{i}\right)^{2} / N}}{\sum_{i=1}^{N} x_{i} / N},
$$

where $x_{i}$ and $x_{i}^{\prime}$ are the $V_{\mathrm{T}}$ of voxel $i$ in the myocardial ROI of the parametric images obtained with the full protocol and with the simplified protocol, respectively, and $N$ is the total number of voxels in the myocardial ROI.

\section{RESULTS}

\section{ROI-Based Kinetic Modeling with Simplified Model}

Figure 1 shows typical fitting results with 2T and LEGA for a sample subject. The fits of the myocardial time-activity curves nearly overlapped. The ROI-based $V_{\mathrm{T}}$ obtained using $2 \mathrm{~T}$ and LEGA are compared in Figure 2. Figure 2A shows that the obtained $V_{\mathrm{T}}$ was almost identical in each of the 2 models. An excellent linear relationship was obtained $(y=0.955 x+0.547$, $\left.R^{2}=0.997\right)$, indicating that LEGA can be used as a simplified
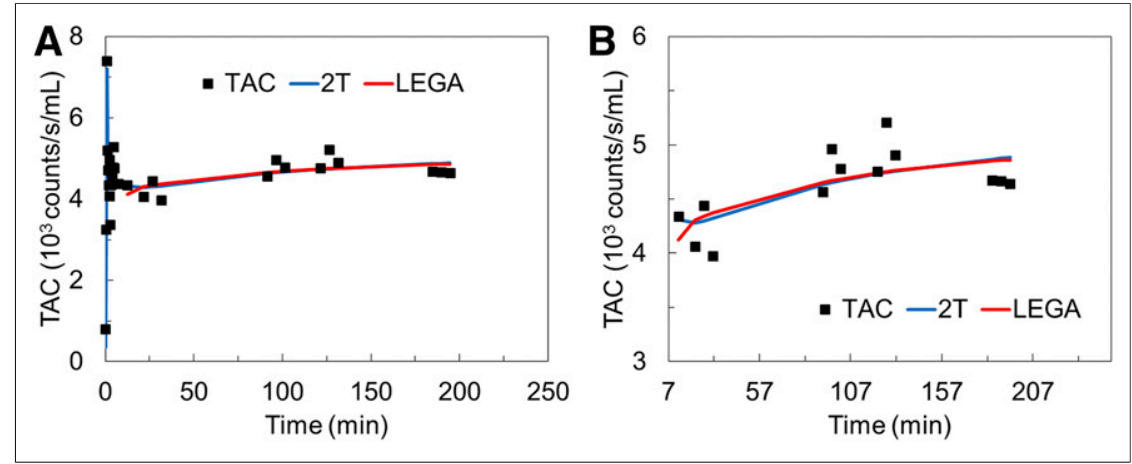

FIGURE 1. Typical time-activity curve (TAC)-fitting results for 2 T and LEGA ( $t^{\star}=7.5 \mathrm{~min}$ ) in healthy human for total of $3 \mathrm{~h}(\mathrm{~A})$ and after $7 \mathrm{~min}(\mathrm{~B})$. model of $2 \mathrm{~T}$ for ${ }^{123} \mathrm{I}-\mathrm{MIBG}$ modeling. The Bland-Altman plot shown in Figure 2B further confirmed this finding. The mean $V_{\mathrm{T}}$ difference between 2T and LEGA was $-0.8 \mathrm{~mL} / \mathrm{cm}^{3}$, and the $95 \%$ limits of agreement were $[-2.5,1.0] \mathrm{mL} / \mathrm{cm}^{3}$, which are small compared with the $V_{\mathrm{T}}$ range of this population $\left(15.0-53.8 \mathrm{~mL} / \mathrm{cm}^{3}\right.$; mean, $\left.29.0 \pm 12.4 \mathrm{~mL} / \mathrm{cm}^{3}\right)$. Although a negative slope $(-0.044)$ significantly different from zero $(P=0.04)$ was observed in the BlandAltman plot, the range of $V_{\mathrm{T}}$ difference $\left(\leq 2.5 \mathrm{~mL} / \mathrm{cm}^{3}\right)$ was small when considering the $V_{\mathrm{T}}$ range $\left(\leq 53.8 \mathrm{~mL} / \mathrm{cm}^{3}\right)$.

\section{ROI-Based Kinetic Modeling with Simplified Protocol}

The ROI-based results using 3 simplified protocols for $2 \mathrm{~T}$ and LEGA are shown in Figure 3 and were compared with the gold standard $V_{\mathrm{T}}$, obtained with the full protocol with 5 scans and 2T. By using $2 \mathrm{~T}$, there was no apparent correlation of $V_{\mathrm{T}}$ between the simplified protocols and the full protocol. In contrast, strong correlations of $V_{\mathrm{T}}$ could be obtained using LEGA, indicating that LEGA is more robust than $2 \mathrm{~T}$ for the simplified protocols when analyzing less dynamic data with large gaps in between. The results showed that the protocol using only 2 scans with a shorter total acquisition time (45 min) is feasible for dynamic ${ }^{123}$ I-MIBG imaging using LEGA. Among these 3 simplified protocols, the $V_{\mathrm{T}}$ results were more reliable when there was a longer interval between the 2 scans, because the $R^{2}$ values derived from the " $30+180 \mathrm{~min}$ " $(0.931)$ and "30 + 120 min" (0.937) protocols appeared to be higher than the $R^{2}$ value derived from the " $30+90$ min" protocol (0.814). However, no significant difference in $R$ values (square root of $R^{2}$ ) was found between the " $30+180 \mathrm{~min}$ " and " $30+120 \mathrm{~min}$ " protocols $(P=0.9)$ or between the "30 $+180 \mathrm{~min}$ " and "30 + $90 \mathrm{~min}$ " protocols $(P=0.1)$ using the Williams' 2 -tailed test (23). The slope of the "30 + 180 min" protocol was closer to $1(0.885)$ than the slope of the " $30+120$ min" $(0.489)$ or " $30+90 \mathrm{~min}$ " $(0.640)$ protocol, with a significant difference, since the linear regression slope between $V_{\mathrm{T}}$ difference of the 2 simplified protocols and the gold standard $V_{\mathrm{T}}$ was significantly different from zero $(P=0.0008$ between " $30+180 \mathrm{~min}$ " and " $30+120 \mathrm{~min}$ "; $P=0.03$ between " $30+180$ min" and "30 + 90 min"). Figure 4 shows the BlandAltman plots between the simplified protocols with LEGA and the gold standard, which confirmed that the mean $V_{\mathrm{T}}$ difference $\left(-1.7 \mathrm{~mL} / \mathrm{cm}^{3}\right)$ was the smallest using the " $30+180 \mathrm{~min}$ " protocol. In the Bland-Altman plots, the slopes $(-0.67$ and -0.36$)$ of the differences were significantly different from zero $\left(P=3 \times 10^{-6}\right.$ and 0.03 ) for the " $30+120 \mathrm{~min}$ " and " $30+90 \mathrm{~min}$ " protocols, respectively, but such was not the case for the " $30+180$ min" protocol (slope, $-0.088 ; P=0.3$ ). These results indicate that when LEGA is used as a simplified model, the "30 + $180 \mathrm{~min}$ " protocol can be selected as an optimized simplified protocol for a dynamic ${ }^{123}$ I-MIBG study in clinical practice.

\section{Parametric Imaging}

Sample $V_{\mathrm{T}}$ image of a healthy human with LEGA using the full protocol is shown in Figure 5 and compared with the 5-min static SPECT image at $3 \mathrm{~h}$ after injection. The results show that the LEGA model can generate $V_{\mathrm{T}}$ parametric images with noise levels that are similar to those of static SPECT images, though more counts are included to generate parametric images. 


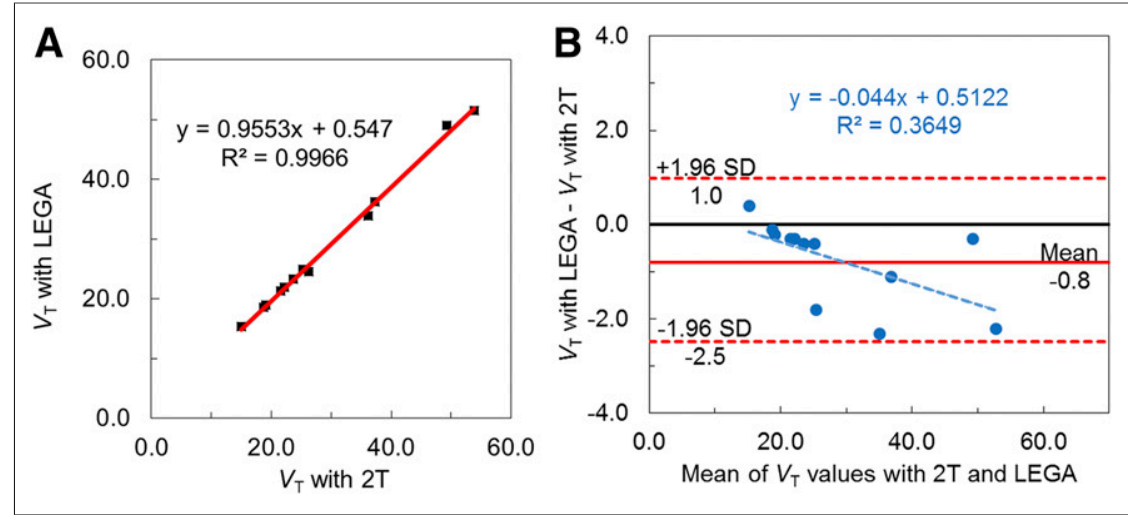

FIGURE 2. Comparison of ROI-based $V_{T}$ obtained using $2 T$ and LEGA: correlation result (A) and Bland-Altman plot (B).

Figure 6 shows the comparison results between the LEGA-derived $V_{\mathrm{T}}$ using ROI-based modeling and the mean myocardial $V_{\mathrm{T}}$ using parametric imaging for the 12 subjects. Figure $6 \mathrm{~A}$ shows a strong linear correlation $\left(y=0.999 x-1.026, R^{2}=0.982\right)$ between the ROI-based and parametric imaging results, demonstrating that parametric imaging of ${ }^{123} \mathrm{I}-\mathrm{MIBG}$ is feasible and accurate. The Bland-Altman plot in Figure 6B shows that the mean $V_{\mathrm{T}}$ difference

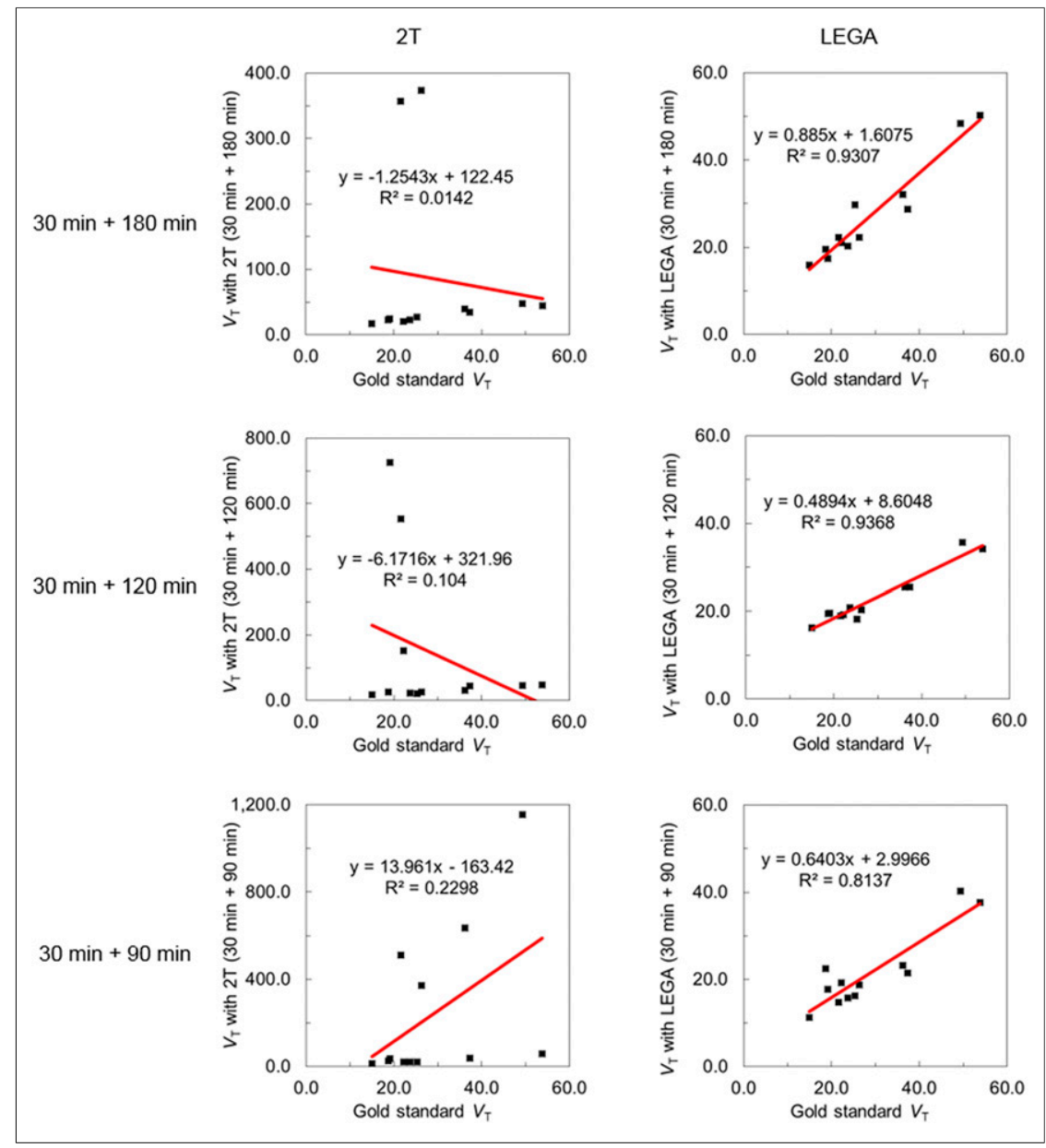

FIGURE 3. Correlation results for ROI-based $V_{\mathrm{T}}$ between gold standard $\left(V_{\mathrm{T}}\right.$ for full protocol with $2 \mathrm{~T}$ ) and simplified protocols $\left(V_{\mathrm{T}}\right.$ for protocols of $30+180 \mathrm{~min}, 30+120 \mathrm{~min}$, and $\left.30+90 \mathrm{~min}\right)$ with both $2 \mathrm{~T}$ and LEGA. between ROI-based and voxel-based analysis was $-1.0 \mathrm{~mL} / \mathrm{cm}^{3}$, and the $95 \%$ limits of agreement were $[-4.2,2.1] \mathrm{mL} / \mathrm{cm}^{3}$, demonstrating consistent $V_{\mathrm{T}}$ quantification. The slope (0.0086) of the differences in the BlandAltman plot was not significantly different from zero $(P=0.8)$.

\section{Parametric Imaging with Simplified Acquisition Protocol}

Sample LEGA $V_{\mathrm{T}}$ images of a healthy human with the simplified protocols are shown in Figure 5 and compared with the $V_{\mathrm{T}}$ image with the full protocol. The $V_{\mathrm{T}}$ image with the " $30+180 \mathrm{~min}$ " protocol was nearly identical to that obtained with the full protocol, whereas the noise level in the $V_{\mathrm{T}}$ images of the other 2 simplified protocols were much higher.

Figure 7 shows the normalized root-mean-squared errors of myocardial $V_{\mathrm{T}}$ between the simplified protocols and the full protocol in parametric images with LEGA calculated by Equation 5 . The mean normalized root-mean-squared error $( \pm \mathrm{SD})$ was $0.24 \pm 0.06$ for the " $30+180 \mathrm{~min}$ " protocol, $0.36 \pm 0.11$ for the " $30+120 \mathrm{~min}$ " protocol, and $0.41 \pm 0.11$ for the "30+ 90 min" protocol. These results indicated a similar conclusion to that made for the ROIbased modeling, where the " $30+180 \mathrm{~min}$ " protocol can be used as an optimized simplified protocol for clinical ${ }^{123}$ I-MIBG parametric imaging using voxel-by-voxel LEGA fitting.

\section{DISCUSSION}

Our study investigated quantitative analysis of ${ }^{123}$ I-MIBG dynamic SPECT images using a simplified model (LEGA) for a healthy human population and compared this simplified model with $2 \mathrm{~T}$, which was identified as the optimal compartment model in our previous study. There was excellent agreement between the $V_{\mathrm{T}}$ obtained from each of the 2 models, indicating LEGA can be used as a simplified model instead of $2 \mathrm{~T}$ in clinical practice. The advantage of using LEGA is that the computation is much faster and more robust to noise than 2T, making LEGA more suitable for implementation in clinical practice. The successful use of a simplified model for analysis of dynamic ${ }^{123}$ I-MIBG SPECT images makes such a model possible for time-consuming and noise-sensitive parametric imaging using voxel-by-voxel fitting. In this study, the parametric image of $V_{\mathrm{T}}$ obtained using LEGA was shown to be of high quality and may provide the possibility for regional analysis of myocardial ${ }^{123}$ I-MIBG uptake in future clinical investigations of the heterogeneity of myocardial sympathetic activation. 

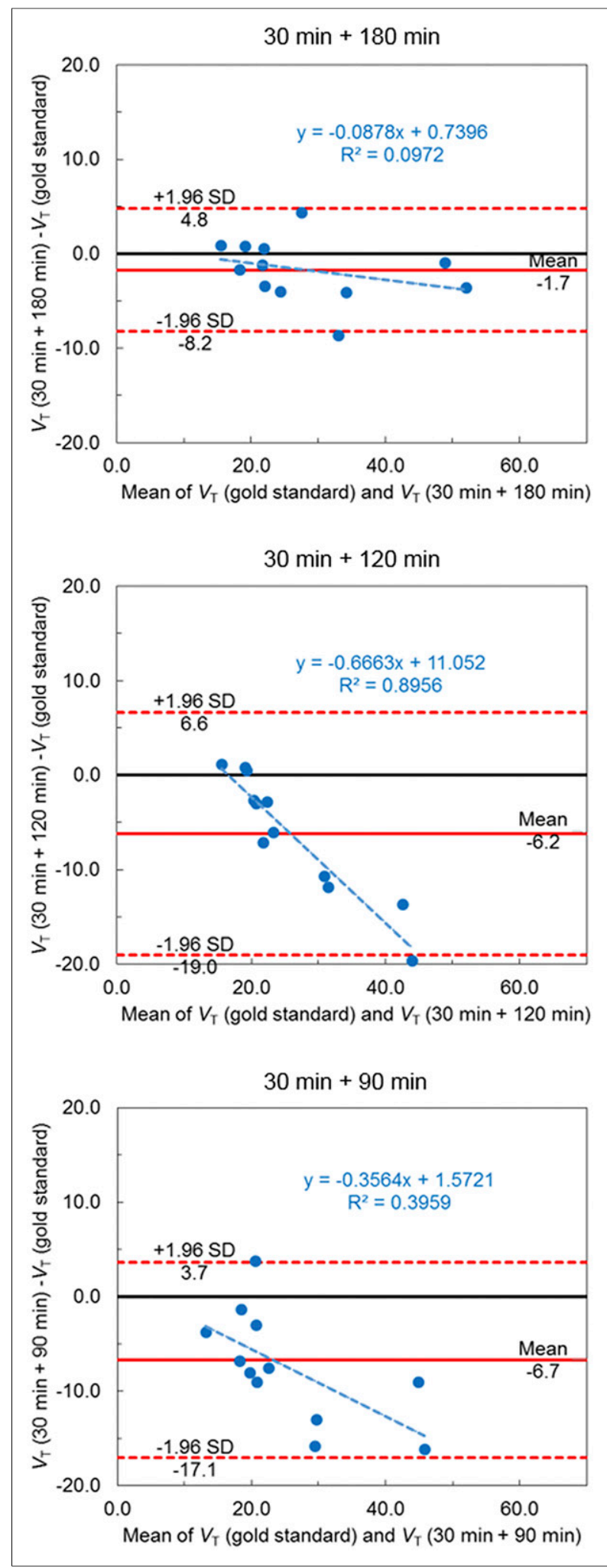

FIGURE 4. Bland-Altman plots of ROI-based $V_{\mathrm{T}}$ between gold standard $\left(V_{T}\right.$ for full protocol with $\left.2 \mathrm{~T}\right)$ and simplified protocols $\left(V_{T}\right.$ for protocols of $30+180 \mathrm{~min}, 30+120 \mathrm{~min}$, and $30+90 \mathrm{~min}$ ) with LEGA.

To implement this ${ }^{123}$ I-MIBG dynamic SPECT imaging technique in clinical practice, simplified acquisition protocols with fewer scans and a shorter total acquisition time were investigated.

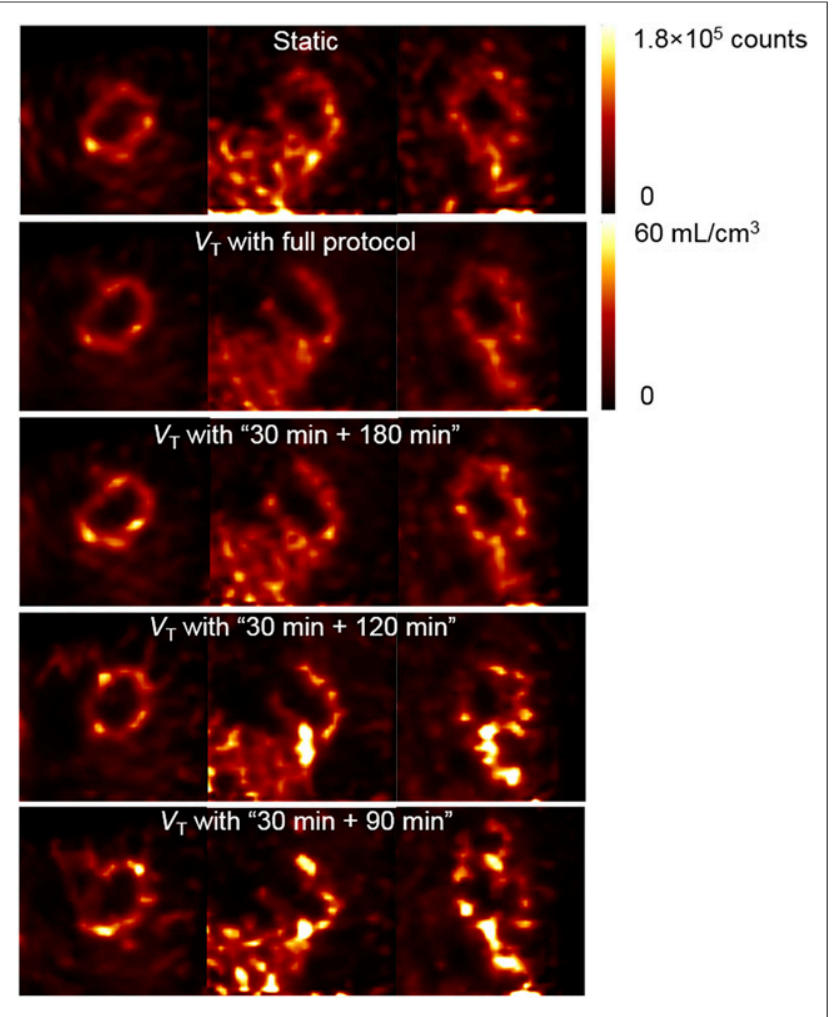

FIGURE 5. Sample 5-min static SPECT image at $3 \mathrm{~h}$ after tracer injection and sample $V_{\mathrm{T}}$ images with LEGA using different protocols for healthy human, shown in transverse, coronal, and sagittal planes. All $V_{\mathrm{T}}$ images were scaled to same maximum.

Three different simplified protocols were investigated using both ROI-based 2T and LEGA analysis. The results indicated that all 3 simplified protocols with $2 \mathrm{~T}$ cannot provide reliable $V_{\mathrm{T}}$. However, the implementation of the simplified protocols was feasible using LEGA. We found that the protocol using an early 30-min acquisition and a delayed 15-min acquisition at $180 \mathrm{~min}$ after injection provided the $V_{\mathrm{T}}$ estimate that best agreed with the gold standard $V_{\mathrm{T}}$ using the full protocol. This finding may indicate that $V_{\mathrm{T}}$ results are more reliable when there is a longer interval between the 2 acquisitions to capture the tracer kinetics. The simulation results reported in the supplemental data (supplemental materials are available at http://jnm.snmjournals.org) show that the $V_{\mathrm{T}}$ results obtained with a delayed scan acquired at 3 , 4 , or $5 \mathrm{~h}$ were quite similar, indicating that the $V_{\mathrm{T}}$ quantification is less sensitive to the exact acquisition time of the delayed scan, as long as it is acquired no earlier than $180 \mathrm{~min}$ after injection. This flexibility makes the proposed simplified protocol easy to implement clinically, because the delayed scan can be acquired whenever the SPECT scanner is available rather than at exactly 4 $\mathrm{h}$ after injection as in the current $2 \mathrm{D}$ heart-to-mediastinum ratio guideline. The $V_{\mathrm{T}}$ images (Fig. 5) indicated that the simplified "30 + 180 min" protocol with voxel-by-voxel LEGA analysis provides a reliable parametric map of $V_{\mathrm{T}}$ for clinical use.

In this study, direct registration between dynamic SPECT images did not provide satisfactory results, because of the noise and truncation artifact in the SPECT images during the parametric imaging process. Alternatively, an attenuation-map-based rigid registration method was implemented to align the dynamic SPECT images to 


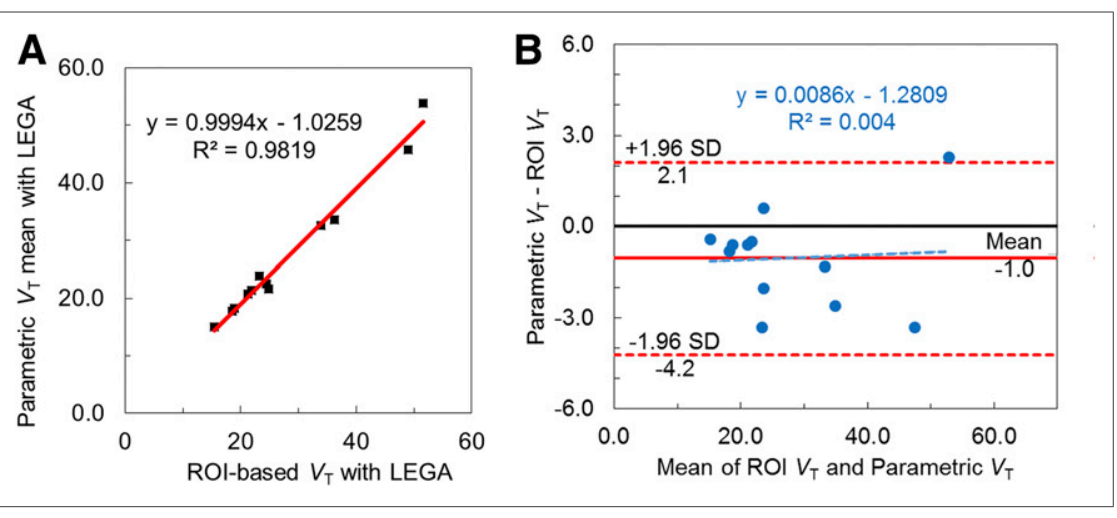

FIGURE 6. Comparison of $V_{\mathrm{T}}$ obtained with LEGA using ROI-based modeling and myocardial $V_{\mathrm{T}}$ obtained with parametric imaging for 12 subjects: correlation result (A) and Bland-Altman plot (B).

\section{CONCLUSION}

Robust simplified quantification of dynamic ${ }^{123}$ I-MIBG SPECT images is feasible with LEGA. A simplified acquisition protocol comprising a 30-min dynamic scan that starts with the ${ }^{123} \mathrm{I}-\mathrm{MIBG}$ injection and a 15-min scan at $180 \mathrm{~min}$ after injection is robust and clinically practical for both ROIbased kinetic modeling and parametric imaging. High-quality parametric $V_{\mathrm{T}}$ images can be obtained for healthy humans with the proposed simplified quantification technique, which might enable regional analysis of ${ }^{123} \mathrm{I}$-MIBG uptake directly from the $V_{\mathrm{T}}$ image in clinical practice. compensate for body motion between scans. However, the body motion is likely to be nonrigid, because the subject left the scanner between scans, though the motion of the heart due to body movement is largely rigid. Our future work will focus on improving the registration accuracy by investigating the nonrigid body motion correction directly using the SPECT data. Respiratory and cardiac motion correction was not used in this study but, if implemented, might further improve the quantification accuracy of the $V_{\mathrm{T}}$ image. Several innovative respiratory and cardiac motion correction methods have been proposed recently (24-28), and we will investigate adding a motion correction technique to our future study protocol.

The simplified quantification technique for analysis of dynamic ${ }^{123}$ I-MIBG SPECT images was validated using a healthy human population in this study. We have also applied the proposed technique to 4 heart failure patients. The results reported in the supplemental data indicate that simplified quantification of dynamic ${ }^{123}$ I-MIBG SPECT is also feasible in these patients. The parametric imaging technique is important because it allows for regional analysis of the patient images and thus may provide a more accurate measurement of $V_{\mathrm{T}}$ when the kinetics differ between the normal and the defect zones of the myocardium. The limitation of this study was the small sample size. A prospective larger trial is needed with more healthy humans and heart failure patients.

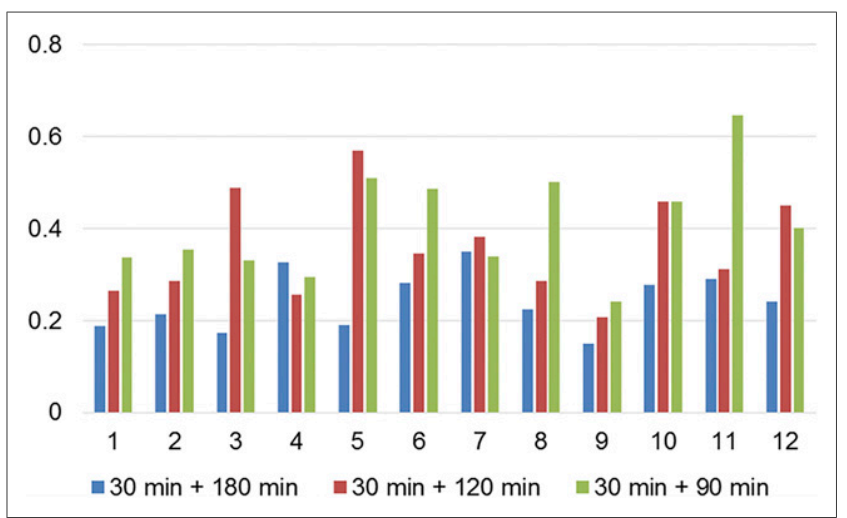

FIGURE 7. Normalized root-mean-squared error of myocardial $V_{\mathrm{T}}$ between simplified protocols and full protocol in parametric images with LEGA for each subject.

\section{DISCLOSURE}

This study was supported by American Heart Association Grantin-Aid awards 13GRNT17090037 and 14GRNT19040010, and research contracts from GE Healthcare. No other potential conflict of interest relevant to this article was reported.

\section{REFERENCES}

1. Travin MI. Cardiac neuronal imaging at the edge of clinical application. Cardiol Clin. 2009;27:311-327.

2. Narula J, Gerson M, Thomas GS, Cerqueira MD, Jacobson AF. ${ }^{123}$ I-mIBG imaging for prediction of mortality and potentially fatal events in heart failure: the ADMIRE-HFX study. J Nucl Med. 2015;56:1011-1018.

3. Nakajima K, Nakata T. Cardiac ${ }^{123}$ I-mIBG imaging for clinical decision making: 22-year experience in Japan. J Nucl Med. 2015;56(suppl):11S-19S.

4. Boogers MJ, Borleffs CJ, Henneman MM, et al. Cardiac sympathetic denervation assessed with 123-iodine metaiodobenzylguanidine imaging predicts ventricular arrhythmias in implantable cardioverter-defibrillator patients. J Am Coll Cardiol. 2010;55:2769-2777.

5. van der Veen BJ, Al Younis I, de Roos A, Stokkel MP. Assessment of global cardiac I-123 MIBG uptake and washout using volumetric quantification of SPECT acquisitions. J Nucl Cardiol. 2012;19:752-762.

6. Chen J, Folks RD, Verdes L, Manatunga DN, Jacobson AF, Garcia EV. Quantitative I-123 mIBG SPECT in differentiating abnormal and normal mIBG myocardial uptake. J Nucl Cardiol. 2012;19:92-99.

7. Lebasnier A, Lamotte G, Manrique A, et al. Potential diagnostic value of regional myocardial adrenergic imaging using ${ }^{123}$ I-MIBG SPECT to identify patients with Lewy body diseases. Eur J Nucl Med Mol Imaging. 2015;42:1043-1051.

8. Dimitriu-Leen AC, Scholte AJ, Jacobson AF. ${ }^{123}$ I-MIBG SPECT for evaluation of patients with heart failure. J Nucl Med. 2015;56(suppl 4):25S-30S.

9. Clements IP, Kelkar AA, Garcia EV, et al. Prognostic significance of ${ }^{123}$ I-mIBG SPECT myocardial imaging in heart failure: differences between patients with ischaemic and non-ischaemic heart failure. Eur Heart $J$ Cardiovasc Imaging. 2016;17:384-390.

10. Yamamoto H, Yamada T, Tamaki S, et al. Prediction of sudden cardiac death in patients with chronic heart failure by regional washout rate in cardiac MIBG SPECT imaging. J Nucl Cardiol. May 12, 2017 [Epub ahead of print].

11. D'estanque E, Hedon C, Lattuca B, et al. Optimization of a simultaneous dualisotope ${ }^{201} \mathrm{Tl} /{ }^{123} \mathrm{I}-\mathrm{MIBG}$ myocardial SPECT imaging protocol with a CZT camera for trigger zone assessment after myocardial infarction for routine clinical settings: are delayed acquisition and scatter correction necessary? J Nucl Cardiol. 2017;24:1361-1369.

12. Prasad R, Thorn S, Chan C, et al. Quantification and kinetic modeling of ${ }^{123} \mathrm{I}-$ mIBG using dynamic SPECT for cardiac innervation [abstract]. J Nucl Med. 2015;56(suppl 3):408.

13. Wu J, Lin SF, Gallezot JD, et al. Quantitative analysis of dynamic ${ }^{123} \mathrm{I}-\mathrm{mIBG}$ SPECT imaging data in healthy humans with a population-based metabolite correction method. J Nucl Med. 2016;57:1226-1232.

14. Logan J, Fowler JS, Volkow ND, et al. Graphical analysis of reversible radioligand binding from time-activity measurements applied to $\left[\mathrm{N}-{ }^{11} \mathrm{C}\right.$-methyl]-(-)-cocaine PET studies in human subjects. J Cereb Blood Flow Metab. 1990;10:740-747. 
15. Carson RE. PET parameter estimation using linear integration methods: bias and variability considerations. In: Uemura K, Lassen NA, Jones T, et al., eds. Quantification of Brain Function: Tracer Kinetics and Image Analysis in Brain PET. Amsterdam, The Netherlands: Elsevier; 1993:499507.

16. Slifstein M, Laruelle M. Effects of statistical noise on graphic analysis of PET neuroreceptor studies. J Nucl Med. 2000;41:2083-2088.

17. Ogden RT. Estimation of kinetic parameters in graphical analysis of PET imaging data. Stat Med. 2003;22:3557-3568.

18. Parsey RV, Ogden RT, Mann JJ. Determination of volume of distribution using likelihood estimation in graphical analysis: elimination of estimation bias. J Cereb Blood Flow Metab. 2003;23:1471-1478.

19. Gallezot JD, Germino MK, Carson RE. Direct EM reconstruction of parametric images from list-mode brain PET using a novel model based on Logan graphical analysis. In: 2016 IEEE Nuclear Science Symposium, Medical Imaging Conference and Room-Temperature Semiconductor Detector Workshop (NSS/MIC/RTSD). Piscataway, NJ: IEEE; 2016.

20. Marquardt DW. An algorithm for least-squares estimation of nonlinear parameters. J Soc Indust Appl Math. 1963;11:431-441.
21. Papademetris X, Jackowski MP, Rajeevan N, et al. BioImage Suite: an integrated medical image analysis suite: an update. Insight J. 2006;2006:209.

22. Bland JM, Altman DG. Statistical methods for assessing agreement between two methods of clinical measurement. Lancet. 1986;1:307-310.

23. Diedenhofen B, Musch J. cocor: a comprehensive solution for the statistical comparison of correlations. PLoS One. 2015;10:e121945.

24. Chan C, Jin X, Fung EK, et al. Event-by-event respiratory motion correction for PET with 3D internal-1D external motion correlation. Med Phys. 2013;40:112507.

25. Chan C, Harris M, Le M, et al. End-expiration respiratory gating for a highresolution stationary cardiac SPECT system. Phys Med Biol. 2014;59:6267-6287.

26. Dasari PK, Konik A, Pretorius PH, et al. Correction of hysteretic respiratory motion in SPECT myocardial perfusion imaging: simulation and patient studies. Med Phys. 2017;44:437-450.

27. Feng T, Wang J, Fung G, Tsui B. Non-rigid dual respiratory and cardiac motion correction methods after, during, and before image reconstruction for $4 \mathrm{D}$ cardiac PET. Phys Med Biol. 2016;61:151-168.

28. Pretorius PH, Johnson KL, King MA. Evaluation of rigid-body motion compensation in cardiac perfusion SPECT employing polar-map quantification. IEEE Trans Nucl Sci. 2016;63:1419-1425. 